\title{
The COSINUS project: perspectives of a NaI scintillating calorimeter for dark matter search
}

\author{
G. Angloher ${ }^{1}$, P. Carniti ${ }^{2}$, L. Cassina ${ }^{2}$, L. Gironi ${ }^{2,3}$, C. Gotti ${ }^{2}$, A. Gütlein ${ }^{4,5}$, D. Hauff ${ }^{1}$, M. Maino ${ }^{2}$, S. S. Nagorny ${ }^{6}$,

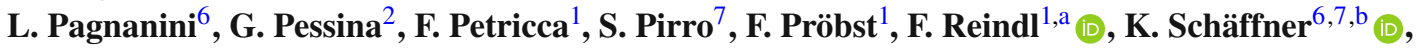 \\ J. Schieck ${ }^{4,5}$, W. Seidel ${ }^{1}$ \\ ${ }^{1}$ Max-Planck-Institut für Physik, 80805 Munich, Germany \\ 2 INFN-Sezione di Milano Bicocca, 20126 Milan, Italy \\ ${ }^{3}$ Dipartimento di Fisica, Università di Milano-Bicocca, 20126 Milan, Italy \\ ${ }^{4}$ Institut für Hochenergiephysik der Österreichischen Akademie der Wissenschaften, 1050 Vienna, Austria \\ ${ }^{5}$ Atominstitut, Vienna University of Technology, 1020 Vienna, Austria \\ ${ }^{6}$ GSSI-Gran Sasso Science Institute, 67100 L'Aquila, Italy \\ ${ }^{7}$ INFN-Laboratori Nazionali del Gran Sasso, 67010 Assergi (AQ), Italy
}

Received: 10 March 2016 / Accepted: 21 July 2016 / Published online: 8 August 2016

(c) The Author(s) 2016. This article is published with open access at Springerlink.com

\begin{abstract}
The R\&D project COSINUS (Cryogenic Observatory for SIgnatures seen in Next-generation Underground Searches) aims to develop a cryogenic scintillating calorimeter using an undoped NaI-crystal as target for direct dark matter search. Dark matter particles interacting with the detector material generate both a phonon signal and scintillation light. While the phonon signal provides a precise determination of the deposited energy, the simultaneously measured scintillation light allows for particle identification on an event-by-event basis, a powerful tool to study materialdependent interactions, and to suppress backgrounds. Using the same target material as the DAMA/LIBRA collaboration, the COSINUS technique may offer a unique possibility to investigate and contribute information to the presently controversial situation in the dark matter sector. We report on the dedicated design planned for the NaI proof-of-principle detector and the objectives of using this detection technique in the light of direct dark matter detection.
\end{abstract}

\section{Introduction}

In the era of precision cosmology we know that dark matter [1] is five times more prevalent than baryonic matter in the Universe, and experimental evidence of dark matter so far solely relies on gravitational interaction.

Among a long list of hypothetical new particles WIMPs (Weakly Interacting Massive Particles) provide compelling

\footnotetext{
a e-mail: florian.reindl@mpp.mpg.de

b e-mail: karoline.schaeffner@lngs.infn.it
}

arguments as this class of candidates, with a mass in the $(\mathrm{GeV}-\mathrm{TeV}) / c^{2}$ region and weak-scale interactions, may provide for a relic density that matches observation. Participation of such particles in weak-scale interactions would also allow for a direct detection in earth-bound detectors via the process of elastic scattering off atomic nuclei [2].

Since the expected recoil energies are in the sub-keV to $\mathrm{keV}$ regime, depending on the mass of the dark matter particle and the target material, the challenge of such direct searches is to combine an ultra-low background with a highly sensitive detection apparatus.

At present, the field of direct dark matter search is very active, with numerous experiments all over the world aiming for further increase in size and sensitivity within the next years.

Direct searches are designed to register signals in the detector induced by the interacting particles. The involved detection channels induced by such scattering processes include light, charge and/or phonon signals. Experiments are either based on a single or on a dual channel readout. Which of the three channels are considered depends on the respective target and the applied detection technique. From the first channel the energy of the interacting particle is determined. In most cases the second channel serves to identify the nature of the interacting particle, thereby providing a powerful tool to discriminate dark matter signals from background.

Dark matter searches may be further divided with respect to the approach they use for detection: a numerous class of experiments is optimized to look for nuclear recoil events in a certain energy window where dark matter particles can induce signals. The number of observed recoil events is con- 
sequently combined with model assumptions, concerning e.g. the density of dark matter, in order to derive properties of dark matter particles, as e.g. their mass and scattering crosssection. Somewhat different therefrom, dark matter may also be identified via an annual modulation signal caused by the seasonal variation of the Earth's velocity with respect to the dark matter halo [3].

At present the situation in the sector of direct dark matter detection is controversial and a definite discovery is still absent. Several experiments [4-6] detect events above the known background level which allow to infer properties of a dark matter particle. In particular, the DAMA/LIBRA collaboration observes in more than 13 annual cycles a statistically robust modulation signal using a total of up to $250 \mathrm{~kg}$ of radiopure sodium iodide $(\mathrm{NaI}(\mathrm{Tl}))$ crystals operated as room temperature scintillating detectors in the Laboratori Nazionali del Gran Sasso (LNGS), a deep underground site in central Italy.

At the same time, the DAMA/LIBRA signal is not consistent, in the standard elastic scattering scenario, with the null results of other direct detection experiments as listed in legend and caption of Fig. 3. However, in order to compare the results of these experiments with the DAMA/LIBRA modulation signal one has to take into account astrophysical assumptions as well as assumptions on the physics of the scattering process of the dark matter particle with the respective target materials. A pure modulation signal instead can be considered model-independent and, thus, has the potential to give a unique and robust signature of new particles. Nonetheless, future investigations are of pivotal importance to understand the origin/composition of the modulation signal observed by the DAMA/LIBRA collaboration either being due to dark matter or a different, so far not understood, physics phenomenon or background.

A series of $R \& D$ projects and experiments with alkali halide crystals as target are presently in construction phase or being upgraded [7-11]. In comparison to the before mentioned scintillation detector approaches, dual channel bolometric detectors using NaI-crystal have some advantages but also face challenges.

\section{Detector design}

The objective of COSINUS (Cryogeni Observatory for SIgnatures seen in Next-generation Underground Searches) is the development of a cryogenic scintillating calorimeter using a NaI-crystal as target - thereby providing a $\mathrm{NaI}$ detector with the possibility for active particle identification on an event-by-event basis thanks to the dual channel detection approach. The use of an alkali halide crystal for a scintillating calorimeter (with dual-channel readout) has already been studied e.g. in $[12,13]$. At first glance, the low Debye temperature of $\mathrm{NaI}$ is not favorable for a detector relying on good phonon propagation properties. However, a thermometer with low heat capacity and high sensitivity as e.g. a transition edge sensor (TES) has a realistic chance to provide a sufficient level of sensitivity $[12,13]$. A major challenge to overcome is the hygroscopic nature of $\mathrm{NaI}$ which asks for a handling of the detector - from the production to the assembling phase - in dry atmosphere using glove box systems and, hence, an adapted production procedure of the detector. Another approach is to coat the fragile NaI-crystal with a protective layer, as was studied by the authors of [14].

While COSINUS will use undoped NaI-crystals, experiments which are only measuring scintillation light typically apply $0.1 \%$ of thallium (Tl) as dopant (e.g. $[6,9-11,15]$ ). However, we want to stress that such small amount of Tldopant does not affect the potential interaction with dark matter, but solely serves to enhance scintillation light output (at room temperature).

Particles scattering in NaI-crystals do not only create phonons, but also scintillation photons. At temperatures of few milli-Kelvin, both the phonon signal in the crystal and the signal arising from the absorption of scintillation photons in a suitable light absorber can be detected by the use of superconducting thin film thermometers: as thin film technology is difficult to be directly applied to the hygroscopic NaI-crystal, a carrier disk made of a more robust scintillating material (e.g. $\mathrm{CdWO}_{4}$ ) is planned to be used instead. The carrier disk (diameter of 40-50 $\mathrm{mm}$ and about $1 \mathrm{~mm}$ thickness) is designed to exceed in diameter the size of the NaI-crystal and carries the thermometer: a transition edge sensor (TES) of CRESST-type consisting of a thin tungsten film $(200 \mathrm{~nm}$, W-TES) directly evaporated onto the carrier crystal. ${ }^{1}$ The target crystals of undoped $\mathrm{NaI}$ can weigh up to $\sim 200 \mathrm{~g}$, depending on the achievable performance in function of the crystal mass. Using a carrier crystal allows to exclude the fragile NaI-crystal from the whole TES production procedures (thin film production by evaporation and sputtering processes, photo-lithography, chemical etching and intermediate cleaning). Once the TES on the carrier crystal is ready, the $\mathrm{NaI}$ is attached to it by a small amount of e.g. silicon oil, as the last step of detector mounting in a dedicated glove box. The delicate NaI-crystal is pressed to the carrier only by its own weight, in this way avoiding the need for metal clamping of the crystal. The phonon propagation from the $\mathrm{NaI}$ to the TES on the carrier occurs via the silicon oil interface.

In order to efficiently reject any $\alpha$-related background, e.g. a recoiling nucleus, which could mimic a dark matter signal, there must not be any non-scintillating surfaces in the line-of-sight of the target crystal. An elegant way to tag and reject such backgrounds is a completely active surround-

\footnotetext{
${ }^{1}$ For optimal resolution and because of their low impedance, TESs are usually read out with SQUID amplifiers [16].
} 
ing of the crystal [17]. Thus in COSINUS, the light detector is planned to consist of a beaker-shaped work-piece made from high purity silicon serving two purposes: scintillation light detection and a fully active surrounding of the target crystal. The beaker will exhibit dimensions of about 40$50 \mathrm{~mm}$ in diameter and height, the wall-thickness will be about $600 \mu \mathrm{m}$. The front face of the polished silicon beaker will be equipped with a W-TES, optimized in size for the purpose of light detection. Despite being quite macroscopic devices, such kind of light detectors have shown to be highly performing, achieving a baseline noise of below $\sigma \simeq 10 \mathrm{eV}^{2}$ [18].

With the help of a dedicated copper structure the target crystal will be held inside the beaker-shaped light detector. Since the carrier crystal is chosen to be slightly larger in diameter than the target crystal and the light detector, an almost $100 \%$ coverage of the target crystal should be achievable. ${ }^{3}$ A schematic drawing thereof can be seen in Fig. 1.

A particle interaction in the target crystal mainly induces a thermal signal detected by the W-TES. This so-called phonon-signal provides a precise measurement of the deposited energy, independent of the type of particle [19,20], often referred to as unquenched channel. ${ }^{4}$ Interactions which take place in the carrier crystal itself can be discriminated from energy deposits in the NaI-crystal by pulse-shape analysis $[18,21]$.

Simultaneously to the phonon signal a small fraction of the energy deposited in the NaI-crystal is emitted in form of scintillation light and detected by the high-purity silicon light-absorbing beaker. The light signal allows to identify the type of interacting particle, as the amount of scintillation light strongly depends on it. Therefore, the light to phonon ratio, referred to as light yield, is characteristic for each type of event. Betas and gammas produce the most light and get assigned a light yield value of one by definition. Other types of particles (e.g. $\alpha$-particles, neutrons inducing nuclear recoils off iodine and sodium) exhibit a lower light yield quantified by the so-called quenching factor [22,23]. The $\mathrm{QF}$ is defined as the ratio of scintillation light produced by an interacting particle of type $X$ to the scintillation light produced by a gamma of the same deposited energy.

The dual channel detection approach of COSINUS aims to allow for a highly efficient discrimination and rejection of background events ( $e^{-} / \gamma \mathrm{s}, \alpha$-particles) from potential signal events (nuclear recoils) in the $\mathrm{NaI}$ target.

\footnotetext{
2 Two beaker-shaped light detectors were operated in CRESST-II phase 2.

${ }^{3}$ In the present set-up there is no line-of-sight to non-scintillating parts (e.g metal clamps of carrier) but only a very small line-of-sight to the scintillation foil surrounding the silicon light absorber.

4 The measured energy in the crystal is independent of the particle, when the small fraction of the energy, escaping in form of scintillating light, is taken into account.
}

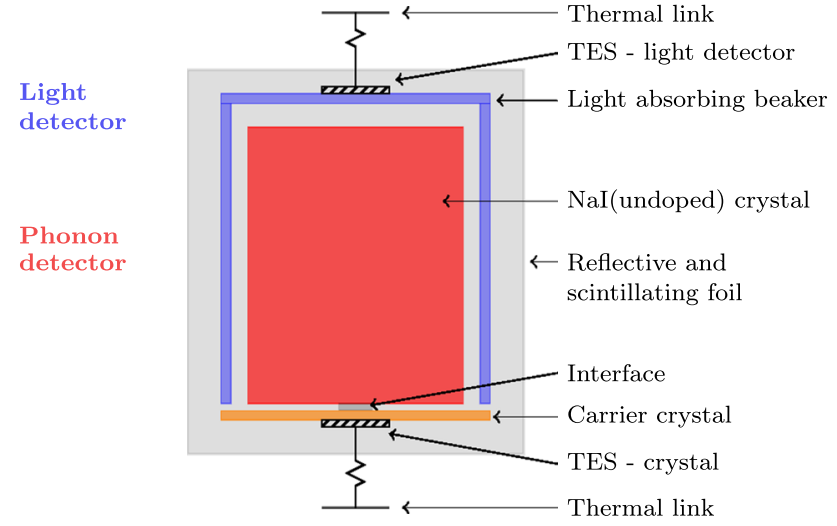

Fig. 1 Concept of a COSINUS detector module consisting of an undoped NaI target crystal and a beaker-shaped light detector. Both detectors, operated at milli-Kelvin temperatures, are read out by transition edge sensors (TES) and are surrounded by a reflective and scintillating foil

The low temperatures required for detector operation will be supplied by a ${ }^{3} \mathrm{He} /{ }^{4} \mathrm{He}$-dilution refrigerator. The test cryostat of the Max-Planck-Institute is the favored choice for all the measurements necessary for the first basic prototype testing. The hall C CUORE/CUPID R\&D dilution refrigerator, since equipped with passive shielding for means of background reduction, is considered for final prototype testing and a longer performance run. Both refrigerators are located in the LNGS underground site for obvious reasons of shielding against cosmic radiation.

The results from CsI measurements give a first reference point on the already achieved performance of an alkali halide crystal operated as cryogenic calorimeter. With a sequence of tests we commissioned the carrier crystal-absorber crystal concept and we achieved, with two different CsI crystals, an energy resolution of $0.95 \mathrm{keV} / 0.70 \mathrm{keV}$, corresponding to a trigger threshold of $4.7 \mathrm{keV} / 3.5 \mathrm{keV}$ [21]. ${ }^{5}$

\section{Performance from simulated data}

We base the performance estimate of a COSINUS detector module on the following assumptions:

- light detector energy resolution from standard performance of silicon beaker light detector operated in CRESST-II phase $2\left(\sigma=0.11 \mathrm{keV}_{e e}\right)$

- phonon detector resolution of $\sigma=0.2 \mathrm{keV}$ (corresponding to a threshold of $5 \sigma=1 \mathrm{keV}$ ),

$-4 \%$ of deposited energy in the NaI detected in the light detector,

\footnotetext{
5 However, a standard CRESST-sized W-TES was used leaving room for future sensitivity improvements via the optimization of the W-TES for the given application.
} 
- a gross exposure (before cuts) of $100 \mathrm{~kg}$-days. Based on results from CRESST-II [19,24], we conservatively estimate an efficiency of $50 \%$ down to an energy of $2 \mathrm{keV}$ and a linear decrease to $20 \%$ efficiency at the threshold of $1 \mathrm{keV}$,

- a flat background contribution down to energy threshold of $1 \mathrm{count} /(\mathrm{keV} \mathrm{kg}$ days) and an additional contribution from ${ }^{40} \mathrm{~K}$ at a level of $600 \mu \mathrm{Bq}$.

In the following the above assumptions will be motivated in more detail.

In COSINUS we aim to bring the performance of a NaIbased detector in line with other scintillating bolometers, rendering an energy resolution of $\sigma=0.2 \mathrm{keV}$, corresponding to an energy threshold of $1 \mathrm{keV}$, feasible. From our understanding the key points to be addressed are the optimization of the sensitivity of the TES itself and of the interface between NaI-crystal and carrier crystal.

In [21] we measured two CsI-crystals; in average around $7 \%$ of the energy deposited in the crystal were determined as scintillation light (for an $\mathrm{e}^{-} / \gamma$-event). From measurements in [13] it is known that CsI roughly emits 3.5 times more energy in form of scintillation light as NaI. However, for COSINUS beaker-shaped light detectors will be used which are found to collect more than twice as much light the light detector of conventional CRESST design used for the measurement in [21]. Thus, in total we expect $7 \% / 3.5 \times 2=4 \%$ of the deposited energy in a NaI-crystal to be detected in the light detector.

Using the above assumptions a simulation is carried out - the outcome is depicted in the light yield-energy plane in Fig. 2 with data points arising from simulated contributions of $\mathrm{e}^{-} / \gamma$-events in black and a potential signal in red. To stick to the DAMA/LIBRA background budget, an overall flat background contribution of $1 \mathrm{count} /(\mathrm{keV} \mathrm{kg}$ days) is assumed.

NaI-crystals typically show a contamination with ${ }^{40} \mathrm{~K}$ which undergoes an electron capture, either directly to the ground state of ${ }^{40} \mathrm{Ar}(0.2 \%$ branching ratio) or to an excited state of ${ }^{40} \mathrm{Ar}$ de-exciting by the emission of an $1.46 \mathrm{MeV}$ gamma ( $10.55 \%$ branching ratio). Both processes are accompanied by the energy releases of 3.2 and $3 \mathrm{keV}$. This contamination makes up for the line at about $3 \mathrm{keV}$ in the $\mathrm{e}^{-} / \gamma$-band in Fig. 2 corresponding to ${ }^{40} \mathrm{~K}$-activity of $600 \mu \mathrm{Bq}$ as measured by the DAMA/LIBRA collaboration [25].

The bands in Fig. 2 are calculated on the basis of the bands determined in the CsI measurement (a detailed description on the underlying model is e.g. given in [22]), the experience of beaker-shaped light detectors operated in CRESST and the assumptions listed above. Thereby, the lines depict the lower and upper $90 \%$-boundaries for the bands corresponding to different event classes to be observed in $\mathrm{NaI}$ (electron recoils in black, nuclear recoils off $\mathrm{Na}$ and $\mathrm{I}$ in

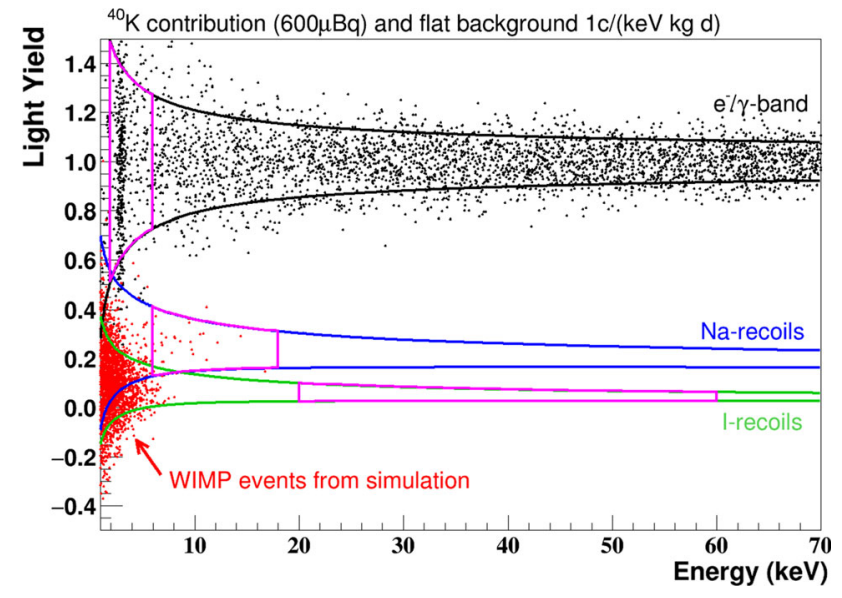

Fig. 2 Simulated data for an exposure before cuts of $100 \mathrm{~kg}$-days applying certain performance assumptions as discussed in detail in the text. The bands correspond to the following event classes: electron recoils in black, scatterings off $\mathrm{Na}$ and I in blue and green. Depicted in black dots are events originating from a flat background contribution of $1 \mathrm{c} /\left(\mathrm{keV} \mathrm{kg}\right.$ days) and a ${ }^{40} \mathrm{~K}$ contamination with an activity of $600 \mu \mathrm{Bq}$. The contribution from ${ }^{40} \mathrm{~K}$ is visible as a line at around $3 \mathrm{keV}$ in the $\mathrm{e}^{-} / \gamma$-band. A hypothetical WIMP scattering off I and $\mathrm{Na}$ nuclei yields the events marked in red. In addition, the magenta boxes indicate the regions which may contribute, apart from the $\mathrm{e}^{-} / \gamma$-band, to the DAMA/LIBRA energy range of positive modulation detection corresponding to 2-6 $\mathrm{keV}_{e e}$

blue and green, respectively). Thus, in between two corresponding lines $80 \%$ of the events of the respective event class are expected. The widths of the bands is dominated by two effects: firstly by the finite baseline resolution of phonon and light detector and secondly by Poissonian fluctuations in the number of scintillation photons produced. The mean of a band obviously depends on the type of particle and the respective quenching factor. For scatterings off $\mathrm{Na}$ and I we use the energy-dependent values reported by Tretyak [23].

In summary, the overall $\mathrm{e}^{-} / \gamma$-background (black dots) is given by the ${ }^{40} \mathrm{~K}$ contamination on top of the constant background level of 1 count/( $\mathrm{keV} \mathrm{kg}$ days). With the performance and backgrounds assumed, we expect five counts below the mean of the Na-band for a gross exposure of $100 \mathrm{~kg}$-days and an energy threshold of $1 \mathrm{keV}$. Thereby, one half of the counts originates from the constant background and the other half from the ${ }^{40} \mathrm{~K}$ contamination, which roughly corresponds to $0.9 \%$ of the activity in the full double-peak. The expected leakage quickly drops with increasing energy, due to the enhanced separation between $\mathrm{e}^{-} / \gamma$-band and Na-band. Additionally, the above numbers show that the leakage is to a large extent caused by the ${ }^{40} \mathrm{~K}$ contamination yielding a considerably diminishing leakage for energies above $3 \mathrm{keV}$, where we expect less than one leakage event (for $100 \mathrm{~kg}$-days before cuts), which is commonly denoted background-free. Considering leakage to the full $80 \% \mathrm{Na}$-band (as depicted in Fig. 2) the values change to 26 events expected above the 


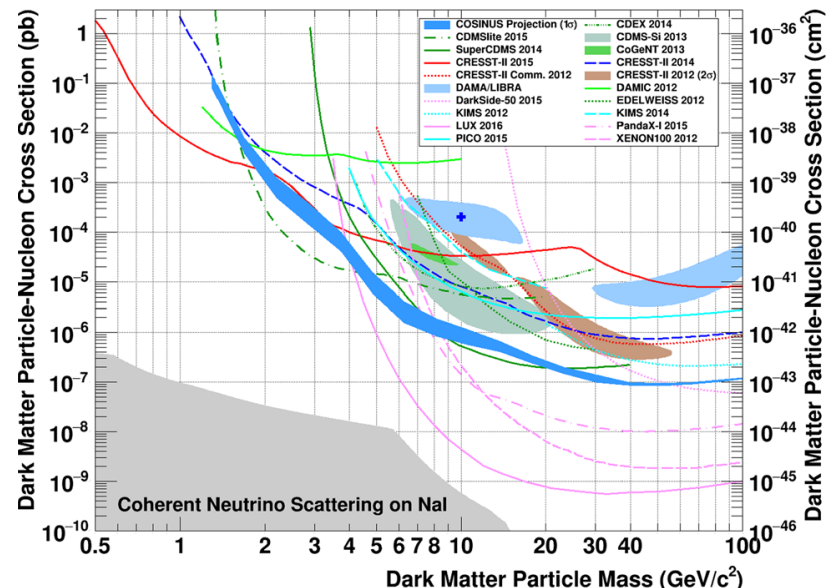

Fig. 3 Projected sensitivity for a NaI-based experiment using COSINUS detector technology [solid blue band, $1 \sigma$ confidence level (C.L.)] for spin-independent elastic dark matter nucleus scattering. Recent results from experiments using silicon and germanium targets are drawn in green [4,28-33], results from CRESST-II $\left(\mathrm{CaWO}_{4}\right)$ are depicted in red $[19,24,34,35]$ and exclusion limits using liquid noble gases in magenta [36-39]. Limits drawn in cyan correspond to bubble chamber technology and experiments with CsI target $[7,8,40]$. The light blue shaded regions correspond to the interpretation of the DAMA/LIBRA $(\mathrm{NaI}(\mathrm{Tl}))$ modulation signal by Savage et al. [27]. The benchmark point (blue cross) indicates the mass and cross-section chosen for the simulated WIMP contribution presented in Fig. 2. Gray-shaded regions in parameter space will be affected by coherent neutrino nucleus scattering on $\mathrm{NaI}$ mainly originating from solar neutrinos [41]

threshold of $1 \mathrm{keV}$, out of which 13 events are attributed to ${ }^{40} \mathrm{~K}$-origin. For the complete Na-band the anticipated leakage drops below one event at $3.9 \mathrm{keV}$.

Because of the high discrimination power we only anticipate a few ten leakage events in the full nuclear recoil bands for the radiopurity levels reported by the DAMA/LIBRA experiment $\left(1 \mathrm{count} /(\mathrm{keV} \mathrm{kg}\right.$ days $\left.)+{ }^{40} \mathrm{~K}: 600 \mu \mathrm{Bq}\right)$, while at the same time expecting $\mathcal{O}\left(10^{3}\right)$ signal events. Hence, we can tolerate a higher level of $\mathrm{e}^{-} / \gamma$-background (as e.g. the two times higher background recently reported for NaIcrystals by the KIMS collaboration [26]), in particular originating from ${ }^{40} \mathrm{~K}$, while still maintaining high sensitivity for a nuclear recoil signal - a distinct feature of COSINUS compared to other NaI-based dark matter searches [6,9-11].

The red events in Fig. 2 result from a contribution of a hypothetical WIMP of $10 \mathrm{GeV} / c^{2}$ and $0.0002 \mathrm{pb}$ which is consistent with the interpretation of the DAMA/LIBRA modulation signal by Savage et al. [27]. The blue-colored islands in Fig. 3 correspond to the DAMA/LIBRA signal regions for scatterings off $\mathrm{Na}$ and I. For clarity we indicated the assumed dark matter particle mass and nucleon cross-section for the simulated data presented here in form of a blue benchmark point (see Fig. 3).

What concerns the distribution of the hypothetical WIMP events we find in the simulation a total of 2386 events in the energy window $1-6 \mathrm{keV}$. About $45 \%$ of these events are present in the energy interval from 1 to $2 \mathrm{keV}$ and about $53 \%$ within $2-6 \mathrm{keV}$. These numbers clearly show the benefit of a low threshold. Despite the conservative assumption on the cut efficiency being $20 \%$ at threshold energy, the number of expected events roughly doubles when lowering the threshold from 2 to $1 \mathrm{keV}$, which is attributed to the anticipated exponential rise of the dark matter recoil spectrum. Above an energy of $6 \mathrm{keV}$ (up to $100 \mathrm{keV}$ ) we find a total of 46 events.

Since WIMPs are expected to scatter coherently off the nucleus as a whole, the cross-section scales quadratically with the atomic mass number $\left(\mathrm{A}^{2}\right)$, thus preferring the heavy I over the light $\mathrm{Na}$. As a consequence, the WIMP events in Fig. 2 appear almost symmetric to the I recoil band. However, the energy transferred in a scattering for the rather light WIMP considered here is enhanced for light nuclei due to kinematic reasons. Consequently, the majority of the 46 events above $6 \mathrm{keV}$ is found in the $\mathrm{Na}$ recoil band. For this reason, cryogenic detectors providing low thresholds in combination with light target nuclei currently lead the field of direct dark matter searches in the low mass regime [24,28].

At last we want to discuss and underline the prominent features of such COSINUS detector in contrast to a purely scintillating detector considering the DAMA/LIBRA experiment as representative. In Fig. 2 we display magenta colored boxes indicating the different regions that may contribute to the positive modulation signal observed by DAMA/LIBRA in an energy window from 2 to $6 \mathrm{keV}_{e e}$. Due to the lack of particle discrimination it remains unknown if the positive modulation signal is made from particles scattering off the electrons or purely off the nuclei in $\mathrm{NaI}(\mathrm{Tl})$.

For interactions with electrons the box extends from 2 to $6 \mathrm{keV}$ ( $\mathrm{e}^{-} / \gamma$-band), the same energy range as for DAMA/LIBRA. Instead, interactions on nuclei are quenched in the light channel, hence the energy interval for $\mathrm{Na}$ and I recoil events has to be corrected for by the respective quenching factor (energy-dependent $\mathrm{QF}$ are taken from [23]). For Na-recoils $(\mathrm{QF} \approx 0.3)$ the region is restricted to about 6 $20 \mathrm{keV}$ as qualitatively indicated by the magenta box in the Na-recoil band. For I-recoils, due to the even higher light quenching effect $(\mathrm{QF} \approx 0.1)$, the box is confined in an energy region of about $20-60 \mathrm{keV}$.

As already mentioned, a distinctive feature of the COSINUS technology is the unquenched phonon channel directly measuring the deposited energy quasi independent of the type of interacting particle. Taking into account the aimed for energy threshold of $1 \mathrm{keV}$, this would result in an improvement in detection threshold by a factor of about six for $\mathrm{Na}-$ recoil events and a factor of about 20 for I-recoils in comparison to the sensitivity demonstrated by DAMA/LIBRA.

It deserves mentioning that we already achieved, by operating two scintillating calorimeters based on CsI an energy threshold as low as 4.7 and $3.5 \mathrm{keV}$, respectively [21]. Thus, 
such detectors already indicate an increased sensitivity for nuclear recoil events in comparison to the DAMA/LIBRA experiment with further refinements anticipated for the future [21].

\section{Perspective and conclusion}

As can be seen, competitive sensitivity also to other direct dark matter searches may be reached, in particular in the mass range of $1-10 \mathrm{GeV} / \mathrm{c}^{2}$ (see Fig. 3). This makes NaI an attractive target for direct dark matter search by itself, beyond offering a material-independent cross-check of the DAMA/LIBRA signal. Of special benefit is the presence of light (Na) and heavy (I) target nuclei which is, however, also given for $\mathrm{CaWO}_{4}$. $\mathrm{A} \mathrm{CaWO}_{4}$-target (CRESST-II/III) offers similar discrimination capabilities and background level, but presumably better phonon properties. The latter are decisive for the achievable energy threshold and resolution rendering $\mathrm{CaWO}_{4}$ better suited for the low-mass range. Currently, NaIcrystals do not reach radiopurity levels of Ge-based experiments (SuperCDMS). This is also true for liquid noble gas experiments (LUX/XENON) which, in addition, are easier to scale to large target masses, but fall behind cryogenic experiments regarding energy resolution and threshold. For masses of $\mathcal{O}\left(\geq 10 \mathrm{GeV} / c^{2}\right)$, however, exposure outweighs the achievable threshold, making liquid noble gases the leading technology in this mass range.

The comparison of results from different experiments, as depicted in Fig. 3, only holds under certain assumptions concerning the dark matter halo and the interaction of dark matter with standard model particles. This consideration is further augmented by the use of different target materials, as the impact of the mentioned uncertainties significantly depends on the target material.

Obviously, material dependencies will be ruled out in the evaluation of COSINUS and DAMA/LIBRA data as both are using $\mathrm{NaI}$ as target. ${ }^{6}$ As Fig. 3 clearly shows, the anticipated sensitivity of a COSINUS detector is about two orders of magnitude below the interpretation of the DAMA/LIBRA claim, assuming a standard dark matter halo and elastic WIMP-nucleus scattering (light-blue regions in Fig. 3 corresponding to recoils off $\mathrm{Na}$ and I, respectively [27]).

The enhanced sensitivity, thereby, is driven by two key factors. Firstly, the particle discrimination via the simultaneous measurement of phonon signal and scintillation. Secondly, the better energy resolution going along with a lower threshold (of $1 \mathrm{keV}$, independent of the type of particle). The latter is of special benefit due to the expected exponential rise of the dark matter recoil spectrum towards low energies.

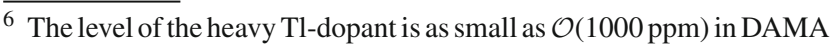
(NaI) [25].
}

Thus, even with a moderate exposure, COSINUS technology has the potential to add knowledge on the underlying nature of the DAMA/LIBRA signal, in particular on the question whether the signal originates from nuclear recoils or not.

For a hypothetical dark matter particle interacting with the electrons of the target material the advantage of particle discrimination vanishes, as the dominant background will also be found in the $\mathrm{e}^{-} / \gamma$-band. This renders radiopurity the key issue, for the experimental set-up and the NaI-crystals. The benefit of an excellent energy resolution and a low threshold still persist. In this scenario a potential future experiment (based on COSINUS technology) would require a higher target mass $(\mathcal{O}(10-100 \mathrm{~kg}))$ measuring for multiple annual cycles. Large-scale bolometric experiments are feasible, as e.g. proven by CUORE [42].

Thus, a potential future dark matter experiment, increased in target mass and based on COSINUS technology, has promising prospects to give new insight on the long-standing DAMA/LIBRA claim.

Acknowledgments This work was carried out in the frame of the COSINUS R\&D project funded by the Istituto Nazionale di Fisica Nucleare (INFN) in the Commissione Scientifica Nazionale 5 (CSN5). In particular, we want to thank the LNGS mechanical workshop team E. Tatananni, A. Rotilio, A. Corsi, and B. Romualdi for continuous and constructive help in the overall set-up construction and M. Guetti for his cryogenic expertise and his constant support.

Open Access This article is distributed under the terms of the Creative Commons Attribution 4.0 International License (http://creativecomm ons.org/licenses/by/4.0/), which permits unrestricted use, distribution, and reproduction in any medium, provided you give appropriate credit to the original author(s) and the source, provide a link to the Creative Commons license, and indicate if changes were made.

Funded by SCOAP ${ }^{3}$.

\section{References}

1. Planck Collaboration, R. Adam et al., Accepted by A\&A (2015). arXiv: 1502.01582

2. M.W. Goodman, E. Witten, Phys. Rev. D 31, 3059 (1985)

3. A.K. Drukier, K. Freese, D.N. Spergel, Phys. Rev. D 33, 3495 (1986)

4. CDMS Collaboration, R. Agnese et al., Phys. Rev. Lett. 111, 251301 (2013). arXiv: 1304.4279

5. CoGeNT Collaboration, C.E. Aalseth et al., Phys. Rev. D 88, 012002 (2013)

6. R. Bernabei et al., Eur. Phy. J. C 73, 2648 (2013). arXiv:1308.5109

7. H.S. Lee et al., Phys. Rev. D 90, 052006 (2014). arXiv: 1404.3443

8. KIMS Collaboration, S.C. Kim et al., Phys. Rev. Lett. 108, 181301 (2012)

9. E. Shields, J. Xu, F. Calaprice, Phys. Procedia 61, 169 (2015). 13th International Conference on Topics in Astroparticle and Underground Physics, TAUP 2013

10. J. Amaré et al., AIP Conf. Proc. 1672, 100001 (2015)

11. DM-Ice Collaboration, J. Cherwinka et al., Phys. Rev. D 90, 092005 (2014)

12. K. Schäffner et al., J. Low Temp. Phys. 167, 1075 (2012)

13. P. Nadeau et al., Astropart. Phys. 67, 62 (2015) 
14. N. Coron et al., Astropart. Phys. 47, 31 (2013)

15. K. Kim et al., Astropart. Phys. 62, 249 (2015)

16. F. Pröbst et al., J. Low Temp. Phys. 100, 69 (1995)

17. R. Strauss et al., Eur. Phys. J. C 75, 1 (2015)

18. F. Reindl et al., in Astroparticle, Particle, Space Physics and Detectors for Physics Applications-Proceedings of the 14th ICATPP Conference, vol. 8, p. 290 (2014)

19. CRESST Collaboration, G. Angloher et al., Eur. Phys. J. C 74, 3184 (2014). arXiv:1407.3146

20. C. Arnaboldi et al., Astropart. Phys. 34, 143 (2010). arXiv: 1005.1239

21. G. Angloher et al., (2016). arXiv:1602.08884

22. R. Strauss et al., Eur. Phys. J. C 74, 1 (2014)

23. V. Tretyak, Astropart. Phys. 33, 40 (2010)

24. CRESST Collaboration, G. Angloher et al., Eur. Phys. J. C 76, 1 (2016)

25. R. Bernabei et al., Nuclear instruments and methods in physics research section A: accelerators. Nucl. Instrum. Meth. A 592, 297 (2008)

26. P. Adhikari et al., Eur. Phys. J. C 76, 1 (2016)

27. C. Savage, G. Gelmini, P. Gondolo, K. Freese, J. Cosmol. Astropart. Phys. 2009, 010 (2009)

28. SuperCDMS Collaboration, R. Agnese et al., Phys. Rev. Lett. 116, 071301 (2016)
29. CDEX Collaboration, Q. Yue et al., Phys. Rev. D 90, 091701 (2014). arXiv:1404.4946

30. SuperCDMS Collaboration, R. Agnese et al., Phys. Rev. Lett. 112, 241302 (2014)

31. CoGeNT Collaboration, C.E. Aalseth et al., Phys. Rev. D 88, 012002 (2013). arXiv: 1208.5737

32. The DAMIC Collaboration, A.A. Aguilar-Arevalo et al., (2013). arXiv: 1310.6688

33. EDELWEISS Collaboration, E. Armengaud et al., Phys. Rev. D 86, 051701 (2012). arXiv: 1207.1815

34. A. Brown, S. Henry, H. Kraus, C. McCabe, Phys. Rev. D 85, 021301 (2012). arXiv:1109.2589

35. G. Angloher et al., Eur. Phys. J. C 72, 1971 (2012)

36. P. Agnes et al., Phys. Lett. B 743, 456 (2015)

37. LUX Collaboration et al., Phys. Rev. Lett. 116, 161301 (2016)

38. PandaX Collaboration, X. Xiao et al., Phys. Rev. D 92, 052004 (2015)

39. XENON100 Collaboration, E. Aprile et al., Phys. Rev. Lett. 109, 181301 (2012)

40. PICO Collaboration, C. Amole et al., Phys. Rev. Lett. 114, 231302 (2015)

41. A. Gütlein et al., Astropart. Phys. 69, 44 (2015). arXiv: 1408.2357

42. D.R. Artusa et al., Adv. High Energy Phys. 2015, 879871 (2015). doi: $10.1155 / 2015 / 879871$ 